\title{
Insights from the Hereditary Thrombotic Thrombocytopenic Purpura Registry: Discussion of Key Findings Based on Individual Cases from Switzerland
}

\section{Hereditary TTP Registry: Günther Landbeck Excellence Award 2019 (Category: Clinical Work)}

\author{
Johanna A. Kremer Hovinga ${ }^{1,2}$ Thomas R. Braschler ${ }^{3}$ Florian Buchkremer ${ }^{4}$ Stefan Farese ${ }^{5}$

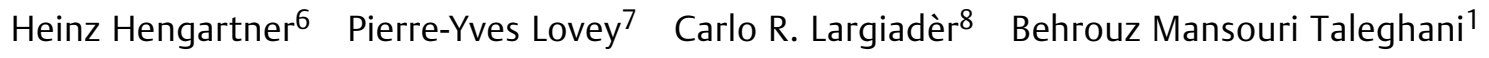 \\ Erika Tarasco ${ }^{1,2}$
}

\footnotetext{
1 Department of Hematology and Central Hematology Laboratory, Bern University Hospital (Inselspital), University of Bern, Bern, Switzerland

${ }^{2}$ Department for BioMedical Research, University of Bern, Bern, Switzerland

${ }^{3}$ Department of Hematology, Luzerner Kantonsspital, Lucerne, Switzerland

${ }^{4}$ Division of Nephrology, Dialysis and Transplantation, Cantonal Hospital Aarau, Aarau, Switzerland

5 Department of Nephrology, Burgerspital, Solothurn, Switzerland

6 Pediatric Hematology-Oncology Unit, Children's Hospital of Sankt Gallen, Sankt Gallen, Switzerland

${ }^{7}$ Service d'hématologie, Hôpital du Valais-Institut Central, Sion, Switzerland

8 University Institute of Clinical Chemistry, Inselspital, Bern University Hospital, University of Bern, Bern, Switzerland

Hämostaseologie 2020;40 (Suppl 1): S5-S14.
}

\author{
Address for correspondence Johanna A. Kremer Hovinga, MD, \\ Department of Hematology and Central Hematology Laboratory, Bern \\ University Hospital, Inselspital, CH-3010 Bern, Switzerland \\ (e-mail: johanna.kremer@insel.ch).
}

Abstract

Keywords
- ADAMTS13
- thrombotic
thrombocytopenic
purpura
- inherited coagulation
disorders
- arterial thrombosis
- pregnancy

The Hereditary TTP Registry is an international cohort study for patients with a confirmed or suspected diagnosis of hereditary thrombotic thrombocytopenic purpura (hTTP) and their family members. Hereditary TTP is an ultra-rare blood disorder (prevalence of $\sim 1-2$ cases per million), the result of autosomal-recessively inherited congenital ADAMTS13 (a disintegrin and metalloproteinase with a thrombospondin type 1 motif, member 13) deficiency (ADAMTS13 activity $<10 \%$ of the normal), and associated with yet many unanswered questions. Until December 2017, the Hereditary TTP Registry had enrolled 123 confirmed hTTP patients. Their median age at disease onset was 4.5 years (range: $0-$ 70) and at clinical diagnosis 16.7 years (range: $0-69$ ), a difference that highlights the existing awareness gap in recognizing hTTP. The systematic collection of clinical data of individual patients revealed their substantial baseline comorbidities, as a consequence of recurring TTP episodes in the past. Most notable was the high proportion of patients having suffered from premature arterial thrombotic events, mainly transient ischemic attacks, ischemic strokes, and to a lesser extent myocardial infarctions. At 40 to 50 years of age and above, more than $50 \%$ of patients had suffered from at least one such event, and many had experienced arterial thrombotic events despite regular plasma infusions every 2 to 3 weeks received

September 20, 2020

accepted

October 5, 2020 (c) 2020 Georg Thieme Verlag KG Stuttgart · New York
DOI https://doi.org/

10.1055/a-1282-2264.

ISSN 0720-9355. 
that supplements the missing plasma ADAMTS13. The article by van Dorland et al. (Haematologica 2019;104(10):2107-2115) and the ongoing Hereditary TTP Registry cohort study were recognized with the Günter Landbeck Excellence Award at the 50th Hemophilia Symposium in Hamburg in November 2019, the reason to present the Hereditary TTP Registry in more detail here.

\section{Introduction}

Hereditary thrombotic thrombocytopenic purpura (hTTP), also known as Upshaw-Schulman syndrome (OMIM \#274150), is a recessively inherited disorder characterized by a severe congenital ADAMTS13 (a disintegrin and metalloproteinase with a thrombospondin type 1 motif, member 13) deficiency (ADAMTS13 activity $<10 \%$ of that in normal plasma) and the consequence of biallelic ADAMTS13 mutations. ${ }^{1-3}$ The ADAMTS13 gene is located on chromosome $9 \mathrm{q} 34$, in close neighborhood of the $A B H$ blood group locus. ${ }^{4-6}$ Today, more than 150 different causative ADAMTS13 mutations, the majority being missense mutations, spreading over all ADAMTS13 protein domains have been identified. ${ }^{7,8}$

Hereditary TTP is rare, and the true prevalence is unknown. Often one to two cases per million have been mentioned. This number fits well for Switzerland with eight (of ten ever diagnosed) hTTP patients currently alive and a permanent resident population of 8.6 million (2019), and also with reported prevalence data for other rare, recessively inherited coagulation disorders. ${ }^{9}$ For Japan and Central Norway, a point-prevalence based on living hTTP cases of 0.4 and 16.7 cases per million has been reported. ${ }^{6,10,11}$ The estimate for Central Norway is in line with the allelic frequencies for the two most prevalent ADAMTS13 mutations, c.4143_4144dupA (p.Gln1382fs) and c.3178C $>T$ (p. Arg1060Trp, p.R1060W) of 0.04 to $0.33 \%$ and 0.3 to $1 \%$ observed in the resident population. ${ }^{6}$ Worldwide, much lower minor allelic frequencies for these two mutations have been reported, approximately $0.08 \%$ for ADAMTS $13 \mathrm{p}$. R1060W (rs142572218) and an even 10-fold lower number for ADAMTS13 c.4143_4144dupA (rs387906343, 0.008\%).

The clinical presentation of hTTP is variable, even within families ${ }^{6,10,12}$ resulting in diagnostic delay and not seldom misdiagnoses, such as neonatal jaundice because of blood group incompatibility, immune-mediated thrombocytopenia (ITP), hemolytic uremic syndrome (HUS), and premature stroke without recognizing the true underlying cause. ${ }^{13-15}$ Clinical presentation of TTP episodes is often less distinct than in immune-mediated TTP with autoantibody-mediated ADAMTS13 deficiency. ${ }^{3,16-19}$ Signs and symptoms of endorgan ischemia that respond to plasma treatment may be present even without (severe) thrombocytopenia and microangiopathic hemolytic anemia. Historically, a dichotomous distribution of age at disease onset had been suggested, ${ }^{13,20,21}$ with patients presenting before the age of 5 years, and a second peak after puberty in early adulthood, often during pregnancy. ${ }^{13,22-24}$ As many of these latter patients underwent exchange transfusions soon after birth, ${ }^{6,10}$ a genuine adult onset can be questioned. But there are also patients, identified during family screenings, who have remained asymptomatic until their 5 th to 7 th decades. ${ }^{10,12}$ However, patients aged 65 years and above are virtually absent in all larger hTTP case series. Whether hTTP patients with milder courses live undiagnosed into advanced adulthood or whether these patients are truly lacking because of premature morbidity and mortality remains to be determined.

Residual ADAMTS13 activity, typically seen in carriers of the c.3178C $>T$ (p.R1060W) mutation, as well as of c.262G $>A$ (p.V88M), was found to partially influence the severity of hTTP..$^{13-15,25}$ Separation according to ADAMTS13 activity levels seems, however, to be less distinct than in hemophilia. The variable clinical courses in patients carrying the same mutation(s) as well as of siblings suggest that there are other yet unknown factors contributing to disease severity. ${ }^{15}$

Acute hTTP episodes are typically treated with plasma infusions; however, more severe bouts and particularly episodes during pregnancy may require larger plasma volumes and hence therapeutic plasma exchange (TPE). ${ }^{3,10,14,15,22,23,26}$ Plasma infusions, administered every 2 (to 3 ) weeks, are effective in preventing acute episodes in many hTTP patients. While a typical and dreaded complication of factor replacement therapy in hemophilia or von Willebrand disease type 3 is the formation of (inhibitory) alloantibodies, this treatment complication seems to be extremely rare in hTTP. To our knowledge, there are only two hTTP patients known so far who have developed low-titer functional ADAMTS13 inhibitors $(<5$ Bethesda unit $/ \mathrm{mL}$ ) while on regular plasma infusions. ${ }^{10,27}$ In a number of additional cases, noninhibitory antiADAMTS13-IgG, usually with fluctuating titers but apparently not interfering with ADAMTS13 recovery or plasma half-life, have been documented. ${ }^{10,17,27}$

The burden of regular plasma infusions is considerable and not all patients are willing or able to subject themselves to such a prophylactic regimen. Guidelines when to start a prophylaxis (i.e. after how many episodes, etc.) or data on long-term benefit of regular plasma infusions are lacking. Here, the Hereditary TTP Registry, initiated in 2006 and since the end of 2012 operating with an electronic database (WebSpirit; 2MT Software GmbH, Ulm, Germany), comes into play. ${ }^{15,28}$ After a decade of data collection, publication of single hTTP cases or small case series addressing specific questions, ${ }^{24,27,29-34}$ and of national projects, $610,35-38$ the first publication on a large patient set was finally achieved in $2019 .{ }^{15}$ We described the key baseline findings of 123 confirmed hTTP patients enrolled until the end of 2017. This publication with the ongoing Hereditary TTP Registry cohort study was recognized with the Günter Landbeck Excellence Award 2019 in the category of clinical work at the 50th 
Hemophilia Symposium in Hamburg, the reason to present the hTTP cohort study in more detail here.

\section{The Hereditary TTP Registry}

The Hereditary TTP Registry (www.ttpregistry.net, ClinicalTrials.gov identifier NCT01257269) was initiated to collect clinical and laboratory data of hTTP patients and their family members. ${ }^{15,28}$ It is set as an ambidirectional cohort study with retrospective data from hTTP patients' birth up to enrolment and then prospective information collected during annual follow-up visits. The goal is to understand the natural history; the long-term course and burden of hTTP; the annual incidence and circumstances of acute hTTP episodes to identify special situations of increased risk; and to understand treatment requirements and treatment-associated side effects. Moreover, we aim at identifying disease modifying and contributing factors. ${ }^{28}$ A community service with expert consultation and advice for physicians treating (possible) hTTP patients is provided and we offer the investigation of all ADAMTS13related laboratory parameters, including analysis of the ADAMTS13 gene, free of charge to study participants and their interested family members from all over the world. In addition, we try to increase awareness for this ultra-rare disorder through presentations at congresses and meetings, by providing educational material on the registry website or through other efforts, such as the Wikipedia articles on UpshawSchulman syndrome in English and German, which were the result of the master thesis of Stutz. ${ }^{39}$

Eligible for participation in the Hereditary TTP Registry are patients with confirmed or suspected hTTP and their family members. The diagnosis of hTTP is established through the following criteria:

1. A suitable patient or family history.

2. Documentation of a severe ADAMTS13 deficiency (ADAMTS13 activity $\leq 10 \%$ of normal) in the absence of a functional ADAMTS13 inhibitor in plasma samples withdrawn at two different time points with an interval of more than 14 days.

3a. Identified homozygous or compound heterozygous ADAMTS13 mutations.

3b. A plasma infusion trial with a full ADAMTS13 recovery and plasma half-life of 2 to 4 days. ${ }^{38,40,41}$

Patients fulfilling criteria 1, 2, and either $3 a$ or $3 b$ are enrolled as confirmed patients. Patients are enrolled as suspected patients when criteria 1 and 2 are met, but molecular analysis of the ADAMTS13 gene lacks, or when only one ADAMTS13 mutation is identified and a plasma infusion trial to assess ADAMTS13 recovery and half-life is lacking. ${ }^{15,28}$ When the results of a pending molecular analysis of the ADAMTS13 gene or of a plasma infusion trial become available, suspected patients can be categorized as confirmed hTTP patients.

At the end of 2019, the Hereditary TTP Registry had 209 participants: 139 confirmed patients, 12 suspected patients (most with pending molecular analysis of the ADAMTS13 gene), and 42 family members, as well as 16 participants identified during workup to have been misdiagnosed (most of them suffering from acquired TTP or ITP). The family members are enrolled (1) to obtain additional information on a patient's disease course, particularly of the neonatal period and infancy through parents, and (2) to study heterozygous ADAMTS13 mutation carriers who typically have an ADAMTS13 activity of about $50 \%$ and are largely regarded as asymptomatic and healthy. ${ }^{10,20}$ Emerging evidence supports the relevance to study heterozygous ADAMTS13 mutation carriers. In the Rotterdam study, people with an ADAMTS13 activity in the lowest quartile (ADAMTS13 activity <70.5\%) were found to have an increased risk of ischemic cardio- and cerebrovascular events and mortality. ${ }^{42-44}$ Also in survivors of an initial acute episode of immune-mediated TTP, reduced ADAMTS13 activity ( $\leq 70 \%$ ) was found to be a risk factor for subsequent ischemic strokes. ${ }^{45}$

At the end of 2019, physicians at 44 sites in 19 countries (on four continents) followed up participants in the Hereditary TTP Registry which has participant information and informed consent documents available in 17 languages. This makes the Hereditary TTP Registry a large international but at the same time a difficult and demanding project, possibly an inevitable aspect when aiming at meaningful patient numbers in an ultra-rare disease.

To provide a feeling for the variable clinical presentations of hTTP, we describe the 10 hTTP patients (of which 8 are alive) diagnosed and living in Switzerland in detail (-Table 1). All, except patient 84.01 (see below), are participants in the Hereditary TTP Registry. We will highlight and compare their individual findings with the overall Hereditary TTP Registry cohort described by van Dorland et al. ${ }^{15}$

\section{Clinical Courses of hTTP Patients Diagnosed and Living in Switzerland}

The brothers 01.01 (born 1964) and 01.04 (born 1975) have been described in detail elsewhere. ${ }^{40,46-48}$ For many years, they have contributed to numerous studies of the Hemostasis Research Laboratory at Bern University Hospital and the surviving brother, patient 01.04, participated in the long-awaited phase I trial on recombinant ADAMTS13 (patient 9). ${ }^{49}$ Their support of our research over the past 25 years was instrumental for the discovery of the von Willebrand factor-cleaving protease, ADAMTS13, and in establishing the link between a severe ADAMTS13 deficiency and TTP. ${ }^{46}$ Patient 01.01 experienced his first TTP episode at the age of 21 years and demonstrated subsequently a course with recurring episodes, presented with a stroke at the age of 25 years, developed a jacksonian epilepsy, had evidence of multiple residues of ischemic cerebral infarctions, and a diagnosis of invalidating psychoorganic syndrome at the age of 32 years. ${ }^{48}$ The latter prevented a prophylactic therapy with regular plasma infusions and the patient died shortly after his 39th birthday because of a massive stroke. His brother experienced four TTP episodes so far, one at 2 and the others at 17 to 21 years of age. Since then he is on regular plasma prophylaxis, receiving two units of plasma ( $\sim 7.5 \mathrm{~mL} / \mathrm{kg}$ body weight) every 3 weeks, and is doing fine. 


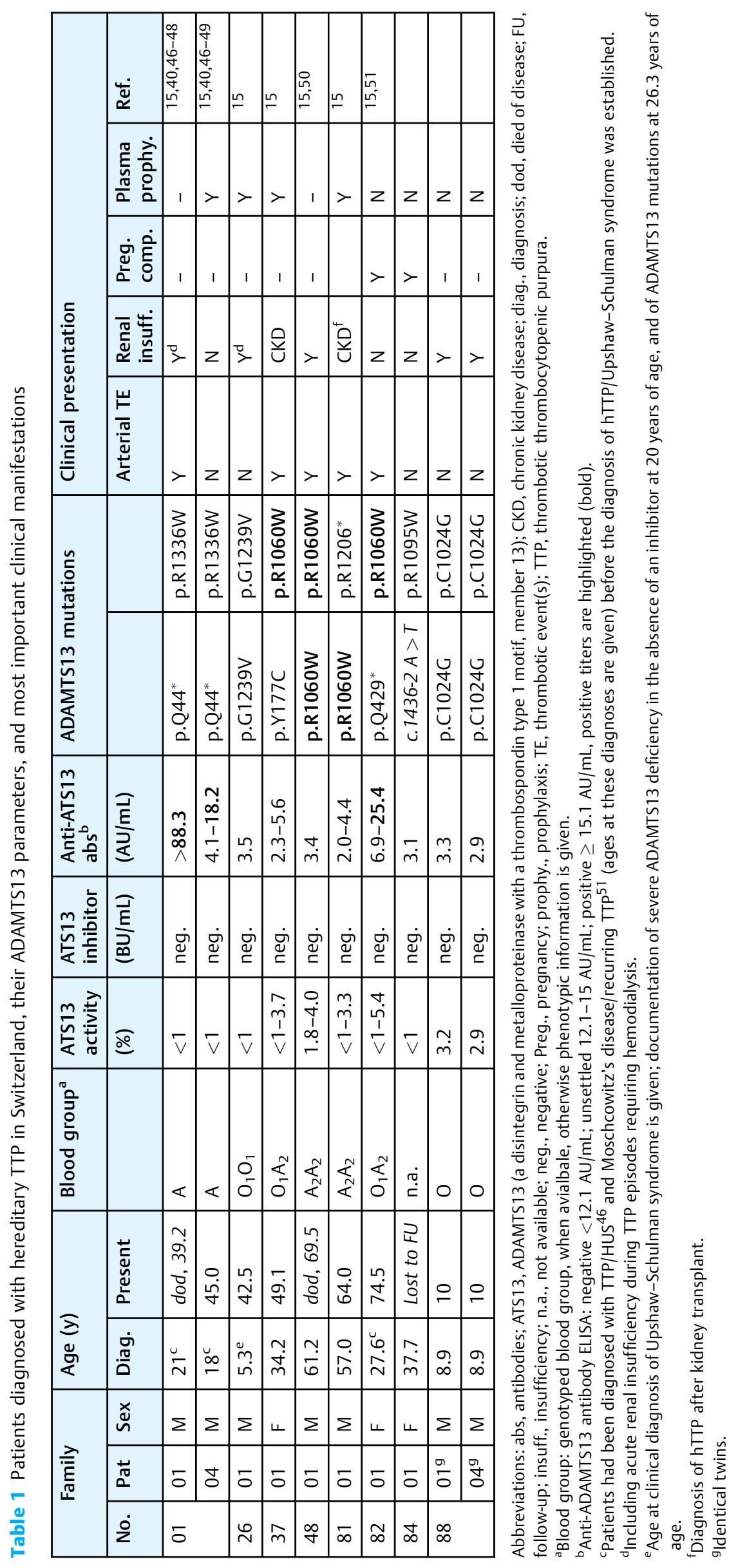


Patient 26.01 (born 1978) originates from a southwestern tributary valley of the Rhone valley in the Swiss Canton of Valais. His parents are unrelated but as the region has been relatively secluded for generations, a common genetic background is probable and explains the homozygous ADAMTS13 mutation (-Table 1). In hindsight, the patient suffered from his first TTP episode at the age of 22.5 months. He was hospitalized with fever and petechial bleeding. ITP was diagnosed and treated with steroids. He subsequently relapsed in the context of fever and pharyngitis, 1 and 4.5 months later. Both episodes resolved spontaneously. At 5 years of age, a fourth acute bout occurred. Diagnosis of TTP, HUS, or a "syndrome similar to the one observed by Upshaw" $^{\prime \prime}$ was considered. Reevaluation of the peripheral blood smears of the previous episodes revealed the presence of schistocytes in episodes one and two. For the first time, treatment included plasma infusions, which had a beneficial effect. For six subsequent episodes, the patient received plasma treatment, either plasma infusions or later in adulthood TPE. From 6 until 17 years of age, the patient was asymptomatic. The first episode as an adult occurred after alcohol intake, the latter provoked three of five acute bouts in adulthood, which were all associated with acute renal failure, requiring hemodialysis in two instances. Prophylactic plasma infusions were administered every 4 weeks for about 2 years, interrupted because of difficulties of reconciling work, family responsibilities, and the burdensome travel to the nearest regional blood center, but restarted after the 10 th acute bout at the age of 29 years, initially with swaying adherence and treatment intervals. Currently, he is receiving two units of plasma every 3 weeks and is doing fine without acute episodes in the past 7 years. His three siblings are asymptomatic, with one sister being a heterozygous ADAMTS13 mutation carrier.

Patient 37.01 (born 1971) was adopted in infancy and little is known about her biological parents. Her mother was heavily addicted and used heroin and possibly alcohol during the patient's pregnancy. Health issues in the neonatal period and thereafter as well as mild developmental and mental disabilities, which became apparent in childhood, were attributed to a neonatal abstinence syndrome and exposure to toxic substances in utero. At the age of 28 years, chronic kidney disease (CKD) was documented and treatment with an angiotensin-converting enzyme inhibitor started. CKD has been relatively stable over the years and the estimated glomerular filtration rate is currently around $60 \mathrm{~mL} / \mathrm{min} / 1.73 \mathrm{~m}^{2}$ (CKD G2$3 a)$. In 2005, she was hospitalized because of a stroke with leftsided hemiparesis due to partial occlusion of the right middle cerebral artery. In addition to this new infarct, there was evidence of prior ischemic lesions in both hemispheres. Laboratory workup revealed a mild thrombocytopenia $\left(113 \times 10^{9} /\right.$ $\mathrm{L}$; first documentation of thrombocytopenia in this patient ever) without schistocytes on the blood smear, a normal hemoglobin $(126 \mathrm{~g} / \mathrm{L})$ and haptoglobin level $(0.39 \mathrm{~g} / \mathrm{L}$, normal range: $0.3-2.0$ ), a slightly increased lactate dehydrogenase $(\mathrm{LDH} ; 715 \mathrm{U} / \mathrm{L}$, normal range $<480)$ and creatinine (111 $\mu \mathrm{mol} / \mathrm{L}$ ). Evaluation for differential diagnoses of thrombocytopenia and hemolysis included an ADAMTS13 activity determi- nation and eventually the diagnosis of hTTP was established. The patient was found to be compound heterozygous for two ADAMTS13 missense mutations, a private mutation in exon 5 (p.Y177C) and the common p.R1060W in exon 24. Regular plasma prophylaxis every 2 weeks was started, with an uneventful course apart from recurring headaches for the next 15 years. Beginning 2020, the patient experienced multiple minor cerebral infarcts, despite fortnightly plasma infusions of $10 \mathrm{~mL} / \mathrm{kg}$ body weight. Clopidogrel replaced aspirin and plasma infusions were administered every week. Because of recurring transient ischemic attacks (TIAs) 1 and 6 months later, temporarily plasma infusions were given twice a week. The presence of an ADAMTS13 allo-antibody was excluded, and a plasma infusion trial demonstrated a full recovery and normal plasma half-life of infused ADAMTS13. At the time of diagnosis with hTTP, however, the patient had documented residual ADAMTS13 activity of about 3\%; in recent years, ADAMTS13 activity before the next plasma infusion was repeatedly $<1 \%$.

Patient 48.01 (born 1945), another male descendant from a second tributary valley of the Rhone valley, was diagnosed with hTTP at the age of 61 years. Since early adulthood a heavy smoker, he underwent endarterectomy for aortoiliac occlusive disease at the age of 42 years, and suffered from an acute inferior-posterior myocardial infarction at the age of 53 years, when arterial hypertension as an additional cardiovascular risk factor was noted. Antihypertensive medication was added to antiaggregant and antiepileptic treatment; the latter had been introduced 2 years earlier because of seizures following a traumatic brain injury. In 2006, he was admitted to a regional hospital with a reduced general state, fever, a productive cough, and dyspnea due to bilateral pneumonia. Despite antibiotic treatment in accordance with the results of the antimicrobial susceptibility testing, the patient's condition deteriorated. Mild anemia and renal insufficiency developed into gross intravascular hemolysis (hemoglobin $57 \mathrm{~g} / \mathrm{L}$ with many schistocytes on the peripheral blood smear) and acute renal failure. The platelet count dropped from $249 \times 10^{9} / \mathrm{L}$ (admission) to $29 \times 10^{9} / \mathrm{L}$ and the patient became confused. Given the high suspicion of a first acute episode of acquired TTP, TPE and glucocorticoids were initiated and clinical remission was achieved within 5 days. Severe ADAMTS13 deficiency, found in a pretreatment sample, confirmed the diagnosis of TTP, although the functional ADAMTS13 inhibitor was negative. While renal function returned to the patient's baseline and complete blood cell count $(\mathrm{CBC})$ was normal during follow-up, severe ADAMTS13 deficiency in the absence of a functional inhibitor or of anti-ADAMTS13 antibodies persisted at 2 and 6 months of follow-up. Molecular analysis revealed the common ADAMTS13 mutation c.3178 C > T, p.R1060W in exon 24 in homozygous state and confirmed hereditary TTP. With the help of a number of family members, we were able to construct the patient's pedigree as far as eight generations back. We identified 172 of 256 potential ancestors with the oldest ancestor born in 1655 . The patient's paternal family had been granted municipal citizenship in the middle of the 15th century in the secluded valley. Although we were unable to formally document consanguinity, there were only seven 
different surnames in the patient's pedigree, and these families had all been registered since the 14 th to mid-16th centuries in this valley. ${ }^{50}$

In the context of a surgical hip replacement, the patient received prophylactic plasma infusions but otherwise refused regular plasma treatment. In 2009, a right-sided sensorimotor stroke with motoric aphasia and dysarthria led to rehospitalization. Computed tomography documented a thrombotic occlusion of the left middle cerebral artery and was successfully treated with intravenous thrombolysis, TPE was initiated a few days later. The patient made a full recovery without neurologic sequelae. Subsequently, medical staff saw him irregularly and again he opted out of prophylactic plasma therapy. His third and final TTP episode occurred 18 months later, triggered by a pneumonia and ongoing considerable alcohol intake. Despite plasma therapy, his condition deteriorated progressively. He died at the age of 69.5 years, 2 months after the onset of pneumonia. His two children, obligatory heterozygous mutation carriers, are healthy at 46 and 49 years of age, respectively.

Patient 81.01 (born 1956) with end-stage renal disease of unknown cause received a cadaveric renal transplant at the age of 56.5 years. Posttransplant renal function deteriorated, a kidney biopsy demonstrated subacute thrombotic microangiopathy, when in addition laboratory findings suggestive of TTP emerged, ADAMTS13 parameters were investigated and eventually confirmed the diagnosis of hTTP at the age of 57 years. Apart from a private nonsense mutation p.R1206*, the patient carries the common p.R1060W mutation. Plasma infusions stabilized the patient's renal function, and then spaced to three to four infusions per year. At the age of 62 years, he experienced a stroke, which was attributed to intermitted atrial fibrillation. Regular plasma infusions every 2 weeks and then every week were started beginning of 2020 because of persistent thrombocytopenia. The latter is typically evidence of platelet clumping in the microcirculation resulting in end-organ ischemia.

The devastating obstetrical history of patient 82.01 (born 1946) has been reported earlier. ${ }^{51}$ In 1973 , aged 27 years at 31 weeks of gestation in her first pregnancy, she presented with severe hypertension $(180 / 110 \mathrm{~mm} \mathrm{Hg})$, edema, proteinuria, acute renal failure, blurred vision, and somnolence that rapidly progressed to coma. Severe preeclampsia was diagnosed and a C-section was performed. The premature twin boys died on days 1 and 4, respectively. The patient's neurological situation remained unchanged; in addition, severe thrombocytopenia $\left(10 \times 10^{9} / \mathrm{L}\right)$, anemia (hemoglobin: $\left.71 \mathrm{~g} / \mathrm{L}\right)$ with numerous schistocytes on the blood smear, and hyperbilirubinemia were noted. Treatment included glucocorticoids, mannitol infusions, unfractionated heparin, several transfusions (not further specified), salicylates, and dipyridamole. Eventually, following a skin biopsy revealing microvascular thrombosis, TTP(Moschcowitz's disease) was diagnosed. Forty-five days after the C-section, the patient underwent relaparotomy for a retroperitoneal abscess and was splenectomized at the same time. Finally, after 6 months of hospitalization, she was transferred to a rehabilitation center for further treatment of her bilateral retinal detachments and right-sided hemiparesis. For months, the $\mathrm{CBC}$ remained abnormal, with platelet counts between 20 and $100 \times 10^{9} / \mathrm{L}$, and a hemoglobin level of 80 to $100 \mathrm{~g} / \mathrm{L}$, and also schistocytes persisted. Following a spontaneous miscarriage at 10 weeks of gestation without further complications, her third pregnancy at the age of 32 years proceeded smoothly until gestational week 31, when again severe preeclampsia manifested, requiring a C-section a few days later. As delivery did not improve the laboratory parameters, recurring TTP was diagnosed. A treatment strategy similar to that in 1973 was followed, with the exception that the patient received whole blood transfusions on the day of the C-section as well as on postoperative days 1,2 , and 9. Two months after the C-section, the patient and her premature but healthy son were discharged. After 6 months, the patient was lost to follow-up until 2013, when she was hospitalized because of a cholecystitis. Thrombocytopenia was noted, which later spontaneously resolved. Given her impressive obstetrical history, ADAMTS13 evaluation was ordered, and an ADAMTS13 activity $<10 \%$ in the absence of an inhibitor was documented on several occasions. Finally, at the age of 67.6 years, the diagnosis of hTTP was established following a molecular analysis of the ADAMTS13 gene, which showed compound heterozygosity for a nonsense mutation, p.Q429*, and the common missense mutation, p.R1060W, typically found in patients with adult-onset hTTP, particularly in pregnancy. ${ }^{13,22,23}$ The patient is in regular follow-up, experienced no further TTP episodes, and is doing fine without regular plasma infusions. Her son currently 42 years of age, has an ADAMTS13 activity of 59\%, in line with the obligatory ADAMTS13 mutation carrier status and is healthy.

Patient 84.01 (born 1977) was admitted at the age of 38 years because of intrauterine fetal death (IUFD) at gestational age $19^{0 / 7}$ weeks associated with gross laboratory abnormalities (platelets $37 \times 10^{9} / \mathrm{L}$; hemoglobin $85 \mathrm{~g} / \mathrm{L}, \mathrm{MCV} 89 \mathrm{fl}$, schistocytes on the blood smear; LDH 1,163 U/L; creatinine $88 \mu \mathrm{mol} / \mathrm{L}$; and normal liver enzymes and INR) in her second pregnancy. Two days earlier, mild hypertension and edema had been noted, as well as fetal growth retardation of about 3 weeks. The placenta showed large infarctions, covering half of the placental surface and histological signs of chronic malperfusion. Given the patient's history of severe preeclampsia and IUFD at gestational age $26^{2 / 7}$ in her first pregnancy 2 years earlier, hTTP was suspected, although the patient's further medical history was empty. A severe ADAMTS13 deficiency in the absence of an inhibitor and of anti-ADAMTS13 antibodies was found on two occasions. Molecular analysis of the ADAMTS13 gene confirmed the suspected hTTP diagnosis with documentation of a splice site and a missense mutation (-Table 1). The patient was lost to follow-up soon thereafter.

Patients 88.01 and 88.04 (identical twin brothers of Congolese origin, born 2010) were known for alpha thalassemia and an attention-deficit hyperactivity disorder. At nearly 9 years of age, patient $\mathbf{8 8 . 0 1}$ was hospitalized because of fever, heavy vomiting, and a rapidly evolving acute renal failure. His brother had presented with a similar picture in the context of a pneumococcal infection 6 months earlier and been diagnosed with an infection-associated HUS. In 88.01, neurological symptoms (Glasgow coma scale of 13-14 
points; slurry speech) were present in addition prompting ADAMTS13 evaluation, which eventually resulted in the diagnosis of hTTP. The boy was monitored one night on the ICU but received no plasma treatment, and the episode resolved spontaneously after 6 days. So far, patient $\mathbf{8 8 . 0 1}$ has experienced three and patient 88.04 two acute TTP episodes, with four of the five acute episodes triggered by upper airway infections and treated with plasma infusions. School entry examinations were normal, although retrospectively the mother reported that the twins were less active, seemed tired, and often complained of headaches during mild infections. Both patients have had cerebral magnetic resonance imaging that were normal and receive plasma infusions on demand when having infections.

\section{The Clinical Course of hTTP Patients in Switzerland in the Light of the Key Findings in 123 hTTP Patients Enrolled in the Hereditary TTP Registry until the End of 2017}

van Dorland et $\mathrm{al}^{15}$ described the main features of the first 123 confirmed patients enrolled into the Hereditary TTP Registry, who had a median age at clinical diagnosis of 16.7 years (range: $0-69$ ), compared to 21.0 years (range: $5.3-$ 61.2 ) in the Swiss subcohort.

Arterial thrombotic events were frequent in all age groups in the Hereditary TTP Registry, with more than $50 \%$ of hTTP patients having suffered from at least one such event at the age of 40 to 50 years and above, particularly frequent were strokes (in $21 \%$ of all patients) and TIAs (in $10 \%) .{ }^{15}$ In the Swiss subcohort, half of the patients had experienced at least one arterial thrombotic event at median 27 years of age (range: $25-$ 64 ), and in four of five patients these events occurred before the age of 50 years. Half of the Swiss hTTP patients developed acute renal failure during acute episodes, while this was seen only in $10 \%$ of the full Hereditary TTP Registry cohort. Infections and pregnancies were the most important triggers of acute TTP episodes in the Swiss subcohort and in the Hereditary TTP Registry, where more than $70 \%$ of patients were on regular plasma prophylaxis. In the Swiss subcohort, only four of the surviving patients currently receive regular plasma prophylaxis, which was implemented in one of them only recently (patient 81.01; at the age of 64 years).

Two of the three Swiss female hTTP patients had their first disease manifestation in the form of severe preeclampsia in their first pregnancy. Their five pregnancies ended with one miscarriage, two intrauterine fetal deaths, and deaths of premature twins in the first days of live. Nowadays, with prophylactic plasma therapy started before or as soon as a pregnancy is confirmed, successful pregnancy outcomes are possible in hTTP patients. $22,23,26$

Interestingly, patients $37.01, \mathbf{4 8 . 0 1}, \mathbf{8 1 . 0 1}$, and 82.01 carry at least one ADAMTS13 allele with the common p.R1060W mutation, which is associated with residual ADAMTS13 activity of about 3 to $5 \%{ }^{13,15,22,23,25}$ In all four patients, we documented at baseline, that is outside of acute episodes, residual ADAMTS13 activities of approximately 3\%. This potential advantage, however, faded away with increasing age and in recent years patients 37.01, 81.01, and 82.01 had ADAMTS13 activities of $<1 \%$. This is in line with the reported decrease of ADAMTS13 activity by $5.7 \%$ per 10 -year increase of age in healthy individuals in the Rotterdam study ${ }^{42}$ and may have contributed to the recent cerebrovascular events in patients 37.01 and 81.01. Residual ADAMTS13 activity was also detected in the twin boys, who are homozygous for another ADAMTS13 mutation in exon 24, p.C1024G that had originally been described by Levy et al. ${ }^{4}$

Regular follow-up of ADAMTS13 parameters revealed in addition fluctuating anti-ADAMTS13-IgG antibody titers in two patients (01.04 and 82.01); their significance is unknown. At least in patient 01.04 who receives regular plasma infusions every 3 weeks, they seem to have no effect on recovery and plasma half-life of infused plasma ADAMTS13. Further characterization of these antibodies is underway.

\section{Discussion and Outlook}

The clinical presentation of hTTP is variable, even within families and hTTP is often not recognized at the first occurrence of disease manifestation, for example, in infancy or during childhood. In our study, we noted that a substantial number of patients had had thrombocytopenia, hemolysis, jaundice, petechiae, and/or anemia during the neonatal period, often leading to therapeutic interventions such as exchange blood transfusion; however, diagnosis of hTTP was established only years later. ${ }^{15}$

Although there are reports on patients who have remained asymptomatic into their 5th to 7th decades, our systematic collection of clinical data revealed substantial comorbidities in hTTP patients. A remarkable finding was the high prevalence of arterial thrombotic events, mainly TIAs and ischemic strokes at a relatively young age. ${ }^{15}$

To prevent futile death and morbidity, $6,10,14,15,24,52,53$ a diagnosis of hTTP should be ruled in or out, respectively, with the evaluation of ADAMTS13 activity particularly in certain specific situations ( - Table 2 ). All newborns with a severe hyperbilirubinemia, especially when concurrent thrombocytopenia is present should have ADAMTS13 activity assessed. Since the introduction of anti-D immunoglobulin prophylaxis in rhesus-negative mothers and the disappearance of rhesus disease, severe neonatal jaundice has become extremely rare and when present should prompt ADAMTS13 testing. This is also true for families in whom newborn babies have died within the first hours to days of life having displayed thrombocytopenia, anemia, and gross hemolysis. ${ }^{53}$ Of note, cord blood can be used for ADAMTS13 activity determination. Although information of normal ranges of ADAMTS13 activity in cord blood and the neonatal period is lacking, values in healthy newborns are typically around 50\% $(30-70 \%) .{ }^{54,55}$

Women presenting with early onset (typically in the second trimester) preeclampsia in their first pregnancy should be evaluated for hTTP. Prophylactic plasma therapy, preexisting or started as early as a pregnancy is confirmed, is able to prevent pregnancy complications in female hTTP patients, and facilitates delivery of healthy infants, at or near term. ${ }^{22,23,26}$ 
Table 2 When to consider a diagnosis of hereditary TTP and initiate ADAMTS13 evaluation

Analysis of ADAMTS13 activity and related parameters-essential in:

- All neonates presenting with severe hyperbilirubinemia, particularly when

- Concurrent thrombocytopenia is present

- An exchange blood transfusion is necessary, or was performed in an older sibling

- An older sibling died in the first days of life with a context of severe hyperbilirubinemia (and thrombocytopenia)

- Women with early severe preeclampsia/HELLP syndrome (occurring typically in the 2nd trimester) in their first pregnancy (and in subsequent pregnancies)

- Patients with premature arterial thrombotic events, primarily strokes and transient ischemic attacks, typically before 40-50 years of age

Analysis of ADAMTS13 activity and related parameters-recommended in:

- Patients experiencing recurring episodes of thrombocytopenia and hemolysis

- That may occur spontaneously

- More often though triggered by infections, alcohol consumption, certain drugs (DDAVP)

- Patients with episodes of malaise and abdominal discomfort, nausea, and vomiting that proceed to (acute) renal failure, especially when concurrent thrombocytopenia and signs of hemolysis are present

Abbreviations: ADAMTS13, a disintegrin and metalloproteinase with a thrombospondin type 1 motif, member 13; HELLP, hemolysis, elevated liver enzyme levels, and low platelet levels; TTP, thrombotic thrombocytopenic purpura.

The third important situation to consider hTTP is in patients presenting with TIA, stroke, or arterial thrombotic events at young age ( $\leq 40-50$ years), particularly when concurrent thrombocytopenia or hemolysis is present. $6,10,14,15,24,52,53$ It should be noted that thrombocytopenia or hemolysis may be mild, in contrast to the usually pronounced thrombocytopenia (and hemolysis) in immunemediated TTP.

Today, the only available treatment for hTTP is plasma infusion (or TPE). Administered every 2 to 3 weeks, plasma infusions prevent acute episodes in many but not all hTTP patients. The decision to start plasma prophylaxis is difficult to take and is associated with an enormous burden, which not all patients are willing to take nor all physicians are yet willing to prescribe. The large number of patients having suffered from premature arterial thrombotic events in the Hereditary TTP Registry, however, suggests that potentially all patients will benefit from a prophylactic regimen, despite the associated burden.

The treatment situation in hTTP is reminiscent to that of hemophiliacs in the 1960s and early 1970s, who were then treated with infusions of fresh blood, plasma, or cryoprecipitates. The prospect of recombinant ADAMTS13, which will potentially be licensed in the next 2 to 3 years, will change treatment of hTTP dramatically and skip 50 to 60 years in comparison with treatment evolution in hemophilia. In these 50-60 years, many research questions were solved, and settled (e.g., on demand vs. prophylactic treatment identification of risk factors for inhibitor development) but also saw the devastating effects of contaminated plasma products. The ever-growing data set of the Hereditary TTP Registry is an excellent tool to address and answer many similar questions.

Recombinant ADAMTS13 will change the life of hTTP patients. Self-administered treatment at home will become possible and likely the standard for most or even all patients in the near future. Until then it is important to identify patients as early as possible to prevent morbidity and mortality. If you care for a patient with a potential diagnosis of hTTP, please contact us. Meaningful data on ultra-rare diseases can only be gained through a collaborative effort.

\section{Funding}

The Hereditary TTP Registry has received support from the Swiss National Science Foundation (grant 310030185233), the Mach-Gaensslen Foundation Switzerland, the ISTH 2007 Presidential Fund, the GTH Congress President Fund, the NFG Foundation, as well as through a research grant from Baxalta US Inc., member of the Takeda group of companies, Bannockburn, Illinois, United States.

\section{Conflict of Interest}

J.A.K.H. is a member of advisory board of Shire, member of the Takeda group of companies and of Ablynx, now part of Sanofi. All honoraria are paid to her employer, Insel Gruppe AG, Bern, Switzerland. The other authors have nothing to declare.

\section{Acknowledgment}

We thank the many sites, the physicians, and patients from all over the world who participate in the Hereditary TTP Registry. We gratefully acknowledge the long-term contribution of the steering committee and the advisory board of the Hereditary TTP Registry.

\section{References}

1 Upshaw JD Jr. Congenital deficiency of a factor in normal plasma that reverses microangiopathic hemolysis and thrombocytopenia. N Engl J Med 1978;298(24):1350-1352

2 Schulman I, Pierce M, Lukens A, Currimbhoy Z. Studies on thrombopoiesis. I. A factor in normal human plasma required for platelet production; chronic thrombocytopenia due to its deficiency. Blood 1960;16:943-957

3 Kremer Hovinga JA, George JN. Hereditary thrombotic thrombocytopenic purpura. N Engl J Med 2019;381(17):1653-1662

4 Levy GG, Nichols WC, Lian EC, et al. Mutations in a member of the ADAMTS gene family cause thrombotic thrombocytopenic purpura. Nature 2001;413(6855):488-494

5 Kokame K, Matsumoto M, Soejima K, et al. Mutations and common polymorphisms in ADAMTS13 gene responsible for von Willebrand factor-cleaving protease activity. Proc Natl Acad Sci U S A 2002;99(18):11902-11907

6 von Krogh AS, Quist-Paulsen P, Waage A, et al. High prevalence of hereditary thrombotic thrombocytopenic purpura in central 
Norway: from clinical observation to evidence. J Thromb Haemost 2016;14(01):73-82

7 Lotta LA, Garagiola I, Palla R, Cairo A, Peyvandi F. ADAMTS13 mutations and polymorphisms in congenital thrombotic thrombocytopenic purpura. Hum Mutat 2010;31(01):11-19

8 Kremer Hovinga JA, Heeb SR, Skowronska M, Schaller M. Pathophysiology of thrombotic thrombocytopenic purpura and hemolytic uremic syndrome. J Thromb Haemost 2018;16(04):618-629

9 Palla R, Peyvandi F, Shapiro AD. Rare bleeding disorders: diagnosis and treatment. Blood 2015;125(13):2052-2061

10 Fujimura Y, Matsumoto M, Isonishi A, et al. Natural history of Upshaw-Schulman syndrome based on ADAMTS13 gene analysis in Japan. J Thromb Haemost 2011;9(Suppl 1):283-301

11 Kokame K, Kokubo Y, Miyata T. Polymorphisms and mutations of ADAMTS13 in the Japanese population and estimation of the number of patients with Upshaw-Schulman syndrome. J Thromb Haemost 2011;9(08):1654-1656

12 Noris M, Bucchioni S, Galbusera M, et al; International Registry of Recurrent and Familial HUS/TTP. Complement factor H mutation in familial thrombotic thrombocytopenic purpura with ADAMTS13 deficiency and renal involvement. J Am Soc Nephrol 2005;16(05):1177-1183

13 Joly BS, Boisseau P, Roose E, et al; French Reference Center for Thrombotic Microangiopathies. ADAMTS13 gene mutations influence ADAMTS13 conformation and disease age-onset in the French cohort of Upshaw-Schulman syndrome. Thromb Haemost 2018;118(11):1902-1917

14 Alwan F, Vendramin C, Liesner R, et al. Characterization and treatment of congenital thrombotic thrombocytopenic purpura. Blood 2019;133(15):1644-1651

15 van Dorland HA, Taleghani MM, Sakai K, et al; Hereditary TTP Registry. The International Hereditary Thrombotic Thrombocytopenic Purpura Registry: key findings at enrollment until 2017. Haematologica 2019;104(10):2107-2115

16 Joly BS, Coppo P, Veyradier A. Thrombotic thrombocytopenic purpura. Blood 2017;129(21):2836-2846

17 Kremer Hovinga JA, Coppo P, Lämmle B, Moake JL, Miyata T, Vanhoorelbeke K. Thrombotic thrombocytopenic purpura. Nat Rev Dis Primers 2017;3:17020

18 Sadler JE. Pathophysiology of thrombotic thrombocytopenic purpura. Blood 2017;130(10):1181-1188

19 Scully M, Cataland S, Coppo P, et al; International Working Group for Thrombotic Thrombocytopenic Purpura. Consensus on the standardization of terminology in thrombotic thrombocytopenic purpura and related thrombotic microangiopathies. J Thromb Haemost 2017;15(02):312-322

20 Furlan M, Lämmle B. Aetiology and pathogenesis of thrombotic thrombocytopenic purpura and haemolytic uraemic syndrome: the role of von Willebrand factor-cleaving protease. Best Pract Res Clin Haematol 2001;14(02):437-454

21 Joly BS, Stepanian A, Leblanc T, et al; French Reference Center for Thrombotic Microangiopathies. Child-onset and adolescent-onset acquired thrombotic thrombocytopenic purpura with severe ADAMTS13 deficiency: a cohort study of the French national registry for thrombotic microangiopathy. Lancet Haematol 2016;3(11):e537-e546

22 Moatti-Cohen M, Garrec C, Wolf M, et al; French Reference Center for Thrombotic Microangiopathies. Unexpected frequency of Upshaw-Schulman syndrome in pregnancy-onset thrombotic thrombocytopenic purpura. Blood 2012;119(24):5888-5897

23 Scully M, Thomas M, Underwood M, et al; Collaborators of the UK TTP Registry. Thrombotic thrombocytopenic purpura and pregnancy: presentation, management, and subsequent pregnancy outcomes. Blood 2014;124(02):211-219

24 Falter T, Kremer Hovinga JA, Lackner K, Füllemann HG, Lämmle B, Scharrer I. Late onset and pregnancy-induced congenital thrombotic thrombocytopenic purpura. Hamostaseologie 2014;34(03): $244-248$
25 Lotta LA, Wu HM, Mackie IJ, et al. Residual plasmatic activity of ADAMTS13 is correlated with phenotype severity in congenital thrombotic thrombocytopenic purpura. Blood 2012;120(02): $440-448$

26 Fujimura Y, Matsumoto M, Kokame K, et al. Pregnancy-induced thrombocytopenia and TTP, and the risk of fetal death, in UpshawSchulman syndrome: a series of 15 pregnancies in 9 genotyped patients. Br J Haematol 2009;144(05):742-754

27 Raval JS, Padmanabhan A, Kremer Hovinga JA, Kiss JE. Development of a clinically significant ADAMTS13 inhibitor in a patient with hereditary thrombotic thrombocytopenic purpura. Am J Hematol 2015;90(01):E22

28 Mansouri Taleghani M, von Krogh AS, Fujimura Y, et al. Hereditary thrombotic thrombocytopenic purpura and the hereditary TTP registry. Hamostaseologie 2013;33(02):138-143

29 Meyer SC, Jeddi R, Meddeb B, Gouider E, Lämmle B, Kremer Hovinga JA. A first case of congenital TTP on the African continent due to a new homozygous mutation in the catalytic domain of ADAMTS13. Ann Hematol 2008;87(08):663-666

30 Deal T, Kremer Hovinga JA, Marques MB, Adamski J. Novel ADAMTS13 mutations in an obstetric patient with Upshaw-Schulman syndrome. J Clin Apher 2013;28(04):311-316

31 Kentouche K, Voigt A, Schleussner E, et al. Pregnancy in Upshaw-Schulman syndrome. Hamostaseologie 2013;33(02): 144-148

32 Bennett M, Chubar Y, Gavish I, Aviv A, Stemer G, Chap-Marshak D. Experiences in a family with the Upshaw-Schulman syndrome over a 44-year period. Clin Appl Thromb Hemost 2014;20(03): 296-303

33 Rank CU, Kremer Hovinga J, Taleghani MM, Lämmle B, Gøtze JP, Nielsen OJ. Congenital thrombotic thrombocytopenic purpura caused by new compound heterozygous mutations of the ADAMTS13 gene. Eur J Haematol 2014;92(02):168-171

34 Fan X, Kremer Hovinga JA, Shirotani-Ikejima H, et al. Genetic variations in complement factors in patients with congenital thrombotic thrombocytopenic purpura with renal insufficiency. Int J Hematol 2016;103(03):283-291

35 Cermáková Z, Hrdliková R, Suláková T, Korístka M, Kovárová $\mathrm{P}$, Hrachovinová I. Thrombotic thrombocytopenic purpura: incidence of congenital form of disease in north Moravia (region Moravia-Silesia). Prague Med Rep 2009;110(03): 239-244

36 von Krogh AS, Kremer Hovinga JA, Tjønnfjord GE, et al. The impact of congenital thrombotic thrombocytopenic purpura on pregnancy complications. Thromb Haemost 2014;111(06): $1180-1183$

37 von Krogh AS, Kremer Hovinga JA, Romundstad PR, et al. ADAMTS13 gene variants and function in women with preeclampsia: a population- based nested case- control study from the HUNT Study. Thromb Res 2015;136(02):282-288

38 Kovarova P, Hrdlickova R, Blahutova S, Cermakova Z. ADAMTS13 kinetics after therapeutic plasma exchange and plasma infusion in patients with Upshaw-Schulman syndrome. J Clin Apher 2019; 34(01):13-20

39 Stutz U. Das Erstellen und Editieren eines Wikipedia-Artikels über das Upshaw-Schulman Syndrom [Master of Medicine]. University of Bern; 2015

40 Furlan M, Robles R, Morselli B, Sandoz P, Lämmle B. Recovery and half-life of von Willebrand factor-cleaving protease after plasma therapy in patients with thrombotic thrombocytopenic purpura. Thromb Haemost 1999;81(01):8-13

41 Fujimura Y, Kokame K, Yagi H, Isonishi A, Matsumoto M, Miyata T. Hereditary deficiency of ADAMTS13 activity: Upshaw-Schulman syndrome. In: Rodgers GM, ed. ADAMTS13: Biology and Disease. Springer International Publishing; 2015:73-90

42 Sonneveld MA, de Maat MP, Portegies ML, et al. Low ADAMTS13 activity is associated with an increased risk of ischemic stroke. Blood 2015;126(25):2739-2746 
43 Sonneveld MA, Franco OH, Ikram MA, et al. Von Willebrand factor, ADAMTS13, and the risk of mortality: the Rotterdam study. Arterioscler Thromb Vasc Biol 2016;36(12):2446-2451

44 Sonneveld MA, Kavousi M, Ikram MA, et al. Low ADAMTS-13 activity and the risk of coronary heart disease - a prospective cohort study: the Rotterdam Study. J Thromb Haemost 2016;14 (11):2114-2120

45 Upreti H, Kasmani J, Dane K, et al. Reduced ADAMTS13 activity during TTP remission is associated with stroke in TTP survivors. Blood 2019;134(13):1037-1045

46 Furlan M, Robles R, Solenthaler M, Wassmer M, Sandoz P, Lämmle B. Deficient activity of von Willebrand factor-cleaving protease in chronic relapsing thrombotic thrombocytopenic purpura. Blood 1997;89(09):3097-3103

47 Antoine G, Zimmermann K, Plaimauer B, et al. ADAMTS13 gene defects in two brothers with constitutional thrombotic thrombocytopenic purpura and normalization of von Willebrand factorcleaving protease activity by recombinant human ADAMTS13. Br J Haematol 2003;120(05):821-824

48 Lämmle B. Clinical problem solving and using new paths in the laboratory: learning from case studies. Hamostaseologie 2020 (epub ahead of print). Doi: 10.1055/a-1191-7198
49 Scully M, Knöbl P, Kentouche K, et al. Recombinant ADAMTS-13: firstin-human pharmacokinetics and safety in congenital thrombotic thrombocytopenic purpura. Blood 2017;130(19):2055-2063

50 Eschler C. Investigation of a Large Valaisan Upshaw-Schulman Syndrome Family [Master of Medicine]. University of Bern; 2014

51 Tapia JE, Hauser GA, Riedler GF. Rezidivierendes, histologisch gesichertes Moschcowitz-Syndrom während des Schwangerschaft. Swiss Med Wkly 1981;111:1287-1292

52 Studt JD, Kremer Hovinga JA, Antoine G, et al. Fatal congenital thrombotic thrombocytopenic purpura with apparent ADAMTS13 inhibitor: in vitro inhibition of ADAMTS13 activity by hemoglobin. Blood 2005;105(02):542-544

53 Hager HB, Andersen MT. A neonate presenting with jaundice, anemia, and thrombocytopenia. Blood 2018;131(14):1627

54 Mannucci PM, Canciani MT, Forza I, Lussana F, Lattuada A, Rossi E. Changes in health and disease of the metalloprotease that cleaves von Willebrand factor. Blood 2001;98(09):2730-2735

55 Studt JD, Kremer Hovinga JA, Alberio L, Bianchi V, Lämmle B. Von Willebrand factor-cleaving protease (ADAMTS-13) activity in thrombotic microangiopathies: diagnostic experience 2001/ 2002 of a single research laboratory. Swiss Med Wkly 2003;133 (23-24):325-332 